\title{
Endometrial cancer with cervical extension in an obese patient: options for surgery versus combined chemoradiotherapy and extra- fascial hysterectomy
}

Correspondence to Dr Pedro T Ramirez, Gynecologic Oncology, MD Anderson Cancer Center, Houston, TX 77030, USA; peramire@mdanderson.org

Accepted 26 April 2019
Check for updates

(C) IGCS and ESGO 2019. № commercial re-use. See rights and permissions. Published by BMJ.

To cite: Kassab C, Ramirez PT, Jhingran A, et al. Int J Gynecol Cancer 2019;29:976-980.

\author{
Presenter: \\ Cynthia Kassab \\ The University of Texas MD Anderson Cancer Center, Houston, Texas, USA \\ Discussant: \\ Pedro T Ramirez \\ Gynecologic Oncology, MD Anderson Cancer Center, Houston, Texas, USA \\ Radiation Oncologist: \\ Anuja Jhingran \\ Radiation Oncology, MD Anderson Cancer Center, Houston, Texas, USA \\ Radiologist: \\ Priya Bhosale \\ The University of Texas MD Anderson Cancer Center, Houston, Texas, USA \\ Pathologist: \\ Russell Broaddus \\ Pathology, MD Anderson Cancer Center, Houston, Texas, USA
}

A 52-year-old $G_{0}$ perimenopausal woman presented with vaginal discharge, in the form of copious amounts of yellow, watery, bloody discharge. As per evaluation performed at an outside institution, the patient was referred to a urologist, for suspected vesicovaginal fistula. Cystoscopy was negative; however, a pelvic computed tomography (CT) scan in April 2018 revealed a $6.7 \mathrm{~cm}$ mass in the cervical canal without any evidence of other disease. She was then referred to the MD Anderson Cancer Center in May 2018 for further evaluation and treatment.

At the time of her presentation, the patient reported persistent discharge and increasing pelvic pressure. Past medical history was significant for dyslipidemia, thyroid disorder, obstructive sleep apnea, and morbid obesity (body mass index 62 $\mathrm{kg} / \mathrm{m}^{2}$ ). Surgical history was significant for total knee arthroplasty. Family history was significant for breast cancer in a maternal grandmother and genitourinary cancer in her maternal grandfather. Pelvic examination revealed normal external genitalia, and speculum examination showed a mass in the cervical os protruding through the endocervical canal. The rectovaginal exam confirmed a barrelshaped cervix extending into the lower uterine segment. Bimanual examination, however, was limited due to patient body habitus. An endometrial biopsy revealed an adenocarcinoma. Immunohistochemistry staining was positive for vimentin, negative for carcinoembryonic antigen, and $30 \%$ positive for estrogen receptors, suggesting an endometrial origin of the tumor. This tumor was therefore considered to be an International Federation of Gynecology and Obstetrics (FIGO) grade 2 endometrial endometrioid adenocarcinoma (Figure 1).

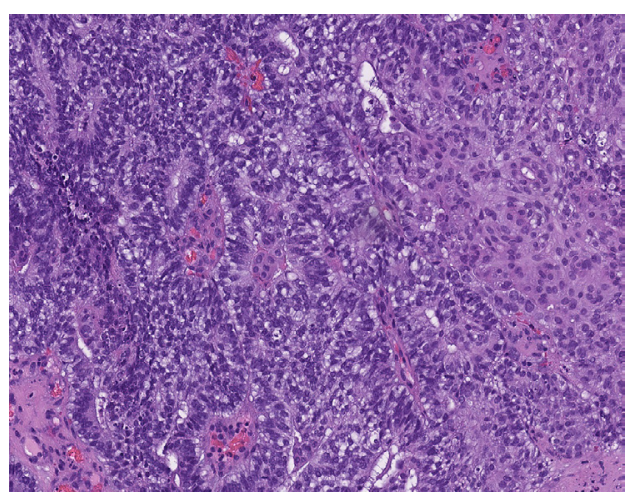

Figure 1 Endometrial biopsy demonstrating moderately differentiated adenocarcinoma (hematoxylin and eosin (H\&E) stain). 


\section{DR BROADDUS}

When considering the differential diagnosis for a mass involving the cervix, a number of potential options arise. These include a primary cervical carcinoma, a primary endometrial carcinoma arising in the fundus and extending to involve the cervix, or a primary endometrial cancer arising in the lower uterine segment and secondarily involving the cervix. Immunohistochemistry performed on the biopsy was most suggestive of an endometrial primary tumor, but we cannot determine whether the tumor was based in the fundus or centered in the lower uterine segment. Lower uterine segment endometrial carcinomas are clinically significant, as approximately $30 \%$ of these tumors have been associated with Lynch syndrome. ${ }^{1}$ Therefore, it is important to perform mismatch repair immunohistochemistry and/or polymerase chain reaction (PCR)-based microsatellite instability analysis as initial steps for evaluation of Lynch syndrome.

\section{DR RAMIREZ}

Based on this information, how would you proceed with further imaging evaluation?

Cervical involvement occurs in approximately $10 \%-15 \%$ of all cases of endometrial cancer. ${ }^{2}$ Therefore, one must be alert to the fact, as in this case, that when a patient presents with an adenocarcinoma after a Pap smear and endometrial biopsy, it is imperative to determine the primary site of disease in order to provide the appropriate recommendation for the patient. When considering the ideal imaging modality, magnetic resonance imaging (MRI) has been shown to be superior to sonography and computed tomography (CT) in determining whether endometrial cancer has invaded the cervix or myometrium. The overall staging accuracy of MRI has been shown to range between $85 \%$ and 93\%. ${ }^{3}$ The reported sensitivity and specificity of MRI in the detection of cervical involvement from endometrial cancer are $72 \%$ and $93 \%$, respectively. The positive and negative predictive values of MRI for detecting cervical involvement from endometrial cancer have been reported to be $90 \%$ and $80 \%$, respectively.

In a study by Ramirez et al, ${ }^{5}$ the authors evaluated the utility of pre-operative MRI in determining whether the primary disease site was the cervix versus the endometrium in patients with inconclusive pre-operative endometrial biopsy. A total of 168 patients were evaluated; and of these, 51 patients met inclusion criteria. The authors found that pre-operative MRI was either inaccurate or inconclusive in $43 \%$ of patients. In a subsequent study by $\mathrm{He}$ et al, ${ }^{6}$ the authors noted that when images were evaluated by dedicated expert gynecologic oncology radiologists, the primary site was identified correctly in $85 \%$ of cases where the endometrial and/or endocervical biopsy was inconclusive. Newer modalities such as positron emission tomography (PET)/CT have been found to have limited sensitivity, specificity, and accuracy in detecting primary lesions. ${ }^{7}$ Therefore, in this patient, we considered that the best and most useful imaging modality in providing better definition of primary disease site was MRI.

\section{DR BHOSALE}

An MRI scan on May 4, 2018 showed a $5.3 \times 5.4 \mathrm{~cm}$ heterogeneous enhancing mass within the cervix. The tumor extended contiguously to the mid-uterus with findings concerning for tumor extension

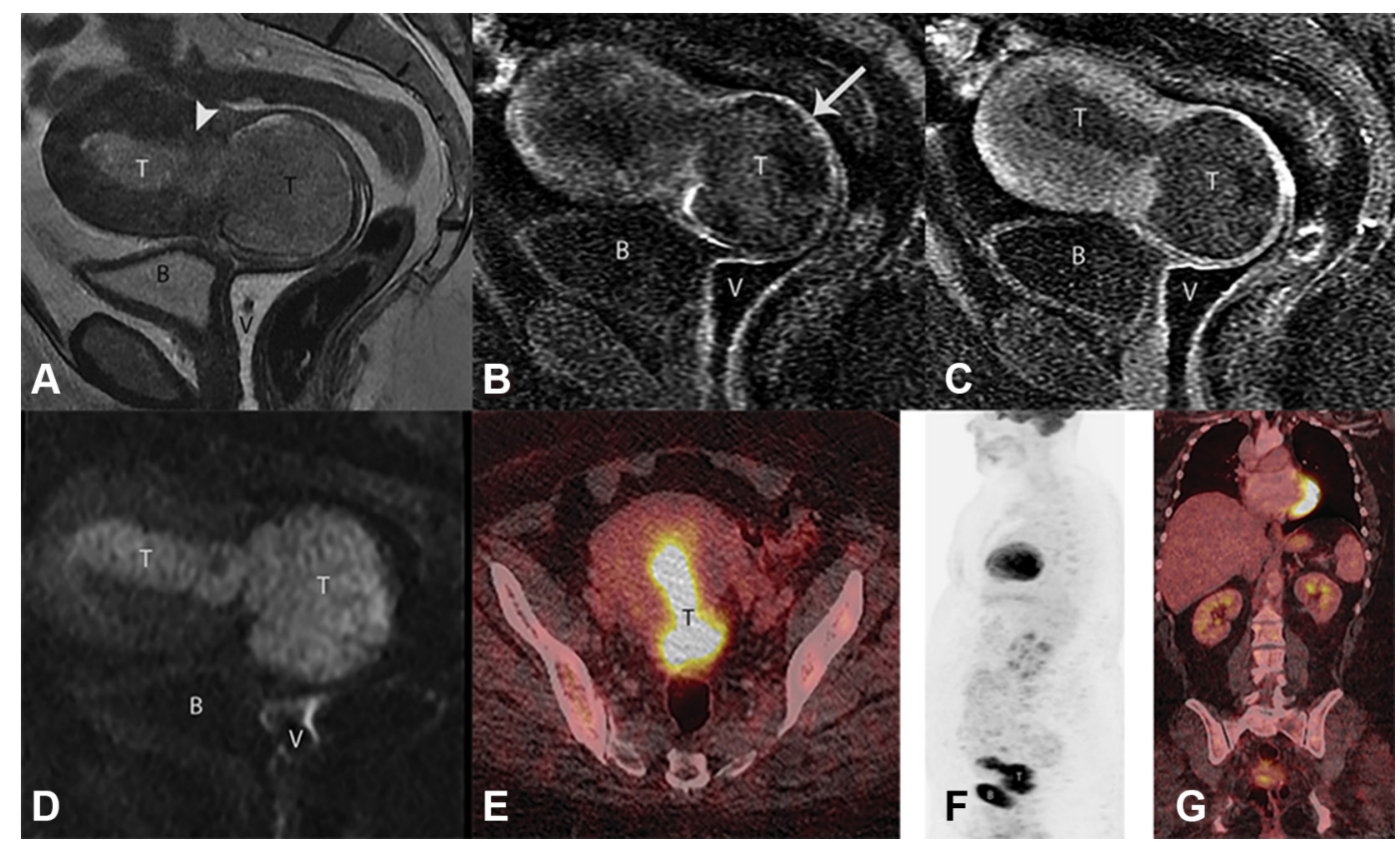

Figure 2 (A) Sagittal T2-weighted image shows an intermediate signal intensity mass (T) in the endometrial cavity extending into the cervix. The cervix is expanded due to the tumor; however, no definite tumor extension into the cervical stroma is noted. There is less than $50 \%$ tumor extension into the posterior uterine wall (arrowhead). (B) Sagittal early contrast-enhanced image demonstrates that the tumor (T) enhances less than the cervix (arrow), suggesting it is an endometrial primary rather than a cervical primary malignancy. (C) Sagittal T1 delayed contrast-enhanced image shows wash out of the tumor (T). (D) Sagittal diffusion sequence with a $B$ value of 600 shows restricted diffusion of the tumor $(T)$. (E) Fused positron emission tomography/ computed tomography (PET/CT) image shows a hypermetabolic mass in the uterus (T). (F) Maximum intensity projection (MIP) image shows a hypermetabolic mass in the uterus (T). (G) Fused PET/CT image shows no distant metastases. B, bladder; V, vagina. 
A

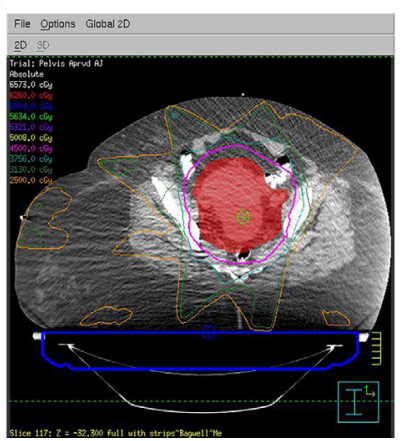

B

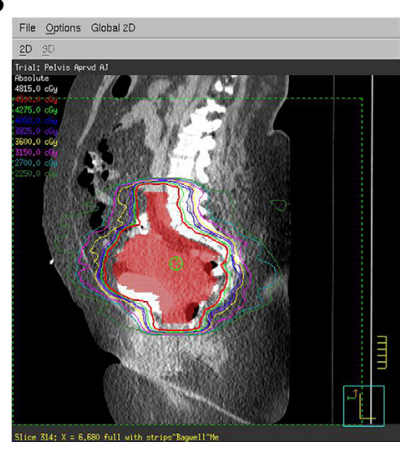

Figure 3 External beam plan using the intensity modulated radiation therapy technique. (A) The axial view shows the uterus in red. The pink line is the 45 Gy line covering the entire uterus. One can see that the entire left side was cutoff on the computed tomography (CT) scan and beams could not be delivered from that side. (B) Sagittal view of the external beam plan with the uterus and cervix in red and the pink line representing 45 Gy.

to the fundus of the uterus. There was no extension to the vagina, bladder, rectum, or parametria. Ovaries contained only bilateral follicles. No pelvic lymphadenopathy was noted, except for non-specific $(1 \mathrm{~cm})$ bilateral common iliac and inguinal nodes. Subsequently, a contrast-enhanced PET/CT scan was performed on May 11, 2018. This showed no supraclavicular, axillary, mediastinal, or hilar adenopathy. There was no evidence of 18F-fluorodeoxyglucose (FDG) uptake in the liver or spleen and there was no retroperitoneal or mesenteric adenopathy noted. There was an FDG-avid mass identified expanding the endometrial canal associated with a large $7 \mathrm{~cm}$ component identified expanding the endocervical canal (Figure 2).

\section{DR RAMIREZ}

\section{Given the MRI findings, what would be the decision process for treatment?}

There are several factors that ought to be considered when proposing a treatment recommendation for this patient. The standard recommendation for a patient with early (stage I-II) endometrial cancer is surgery in the form of total hysterectomy and bilateral salpingo-oophorectomy. However, one needs to determine if the patient is an ideal candidate for surgery and whether a surgical intervention will increase the likelihood of peri-operative morbidity or mortality. According to the 2019 National Comprehensive Cancer Network (NCCN) Guidelines, ${ }^{8}$ for patients with suspected or gross cervical involvement, with endometrioid histology, a cervical biopsy or pelvic MRI should be performed. If there is clear demonstration of cervical stromal involvement, and the patient has no absolute contraindication to surgery, then one may elect one of two options. First, one may opt for surgery in the form of either a total simple hysterectomy or a radical hysterectomy and bilateral salpingo-oophorectomy, along with surgical staging with the consideration of sentinel lymph node mapping in the setting where an established algorithm is routine practice. One should note that prior studies have shown that when comparing type of hysterectomy (simple versus radical) in the setting of stage II endometrial cancer, there were no differences noted in disease-free or overall survival. ${ }^{9}$ Alternatively, one may consider a non-surgical approach by offering the patient external beam radiotherapy plus brachytherapy. When the patient is absolutely

not a candidate for surgery, one may consider either external beam radiotherapy plus brachytherapy, in conjunction with systemic therapy, or opt for systemic therapy alone. In this case, both of these options were considered; however, given the patient's morbid obesity and high risk for peri-operative complications, the decision was made to review the case in a multidisciplinary conference where the ultimate recommendation was discussed among radiation oncologists, gynecologic oncologists, pathologists, and radiologists.

The patient was discussed in the Multidisciplinary Disposition Conference and was subsequently referred for consultation with Radiation Oncology.

\section{DR JHINGRAN}

Please provide details regarding the discussion pertaining to expectations on response and consideration of subsequent post-radiation surgery

In a patient such as this one, we routinely discuss in detail the purpose of the pre-operative radiation therapy with an emphasis on the principle of reducing tumor size and treating the parametrial tissue, thus allowing for the option of a simple hysterectomy at completion of chemotherapy and radiation. Such an approach allows the patient the benefit of avoiding the risk of complications. In offering pre-operative radiation therapy, we would also aim to treat the pelvic lymph nodes, therefore avoiding the need for a subsequent lymph node dissection, equally peri-operative complications. The goal of the radiation therapy is to treat microscopic disease in the lymph nodes as well as to decrease the tumor size in the uterus, and treat the parametrial tissue. However, recognizing that in this setting it would be difficult to achieve an adequate dose to the fundus, the need for subsequent surgery was therefore stressed to the patient.

The patient was recommended treatment in the form of pre-operative radiotherapy with a plan for subsequent extra-fascial hysterectomy. The proposed treatment was with external beam radiation therapy to 45 Gy followed by a 72-hour brachytherapy implant ( $75 \%$ of the dose given for definitive treatment) (Figure $3 A, B)$. In this case, treatment was delivered with low-dose-rate brachytherapy because a larger size tandem was required based on uterine size; however, under circumstances of a normal sized uterus, high dose-rate brachytherapy (usually in three fractions) would have been appropriate. The goal of the brachytherapy was to treat the cervical mass, superior and inferior margins in the proximity of the cervix, as well as the parametrial tissue, but not the entire uterus (Figure 4). In addition, the patient was recommended treatment with concurrent weekly cisplatin $\left(40 \mathrm{mg} / \mathrm{m}^{2}\right)$. The patient developed mild tinnitus during her treatment; however, she did complete a total of five cycles. The patient completed radiotherapy in July 2018. She received a total of 45 Gy in 25 fractions followed by a sixth fraction nodal boost. A pelvic MRI was performed on June 27, 2018 to evaluate disease status prior to brachytherapy.

\section{DR BHOSALE}

Findings revealed evidence of a $2.4 \times 1.5 \mathrm{~cm}$ cervical mass that had decreased in size when compared with the baseline study. No significant pelvic adenopathy was noted (Figure 5). 

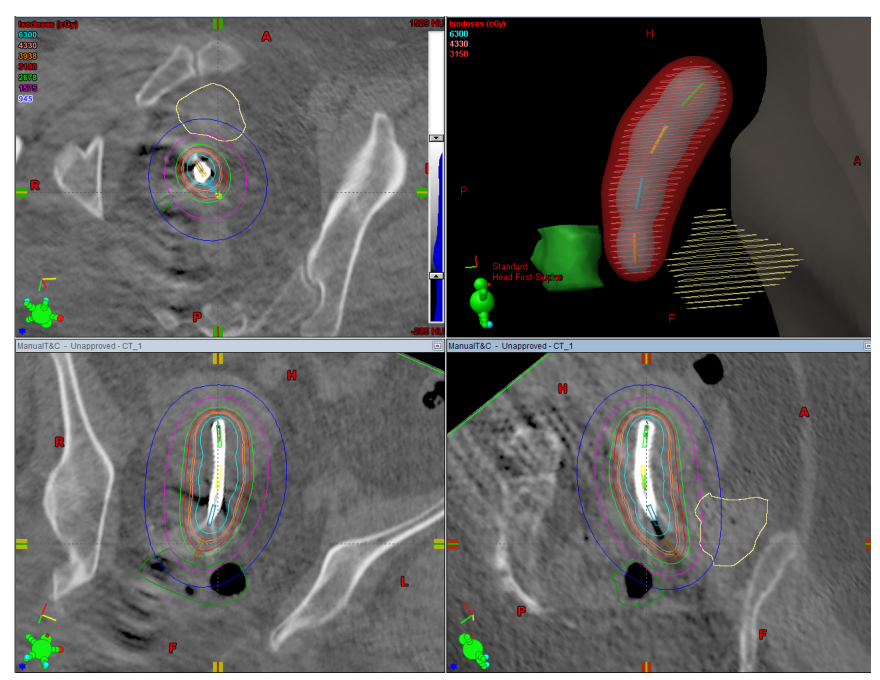

Figure 4 Brachytherapy plan showing four views including axial, sagittal, and coronal views. The brachytherapy implant delivered between 31.5 Gy to the parametrium (total with external beam $76.5 \mathrm{~Gy}$ ) and 43.3 Gy to the entire cervix and some of the uterus (total $88.3 \mathrm{~Gy}$ ), but as can be seen the fundus of the uterus was not covered by the implant. The bladder (in yellow) received between 9 and 15 Gy with the implant (pink and blue lines).

\section{DR RAMIREZ}

At this point, with evidence of residual disease at completion of radiation therapy, what options would you offer the patient?

Historically, the approach of pre-operative radiation therapy with a combination of external beam radiation and low dose-rate (LDR) brachytherapy to doses of 60-70 Gy prescribed to point $A$ was a standard approach in patients with endometrial cancer and significant co-morbidities. ${ }^{10}$ More recently, there has been

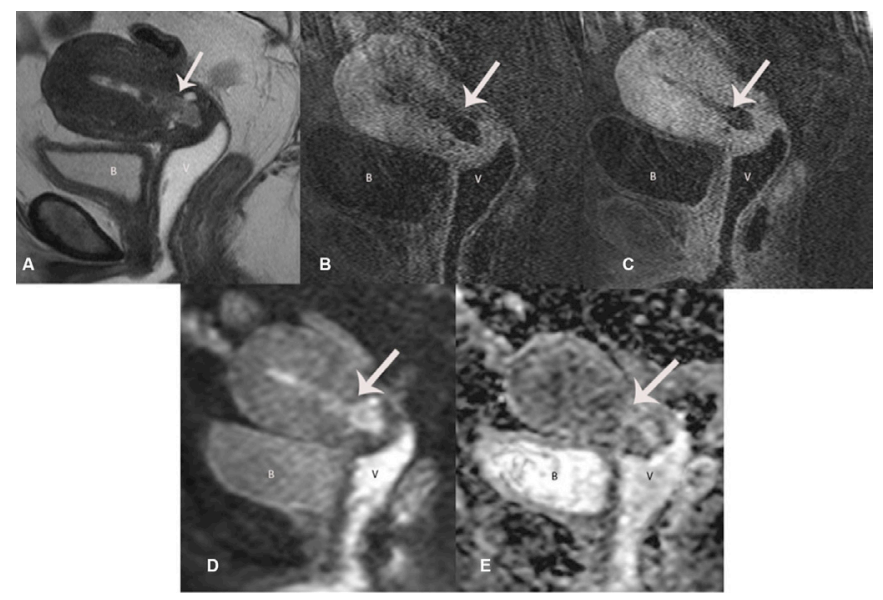

Figure 5 Post-treatment magnetic resonance imaging (MRI) shows a mass-like area in the lower uterine segment and the cervix (arrow). The mass has a high signal on the T2 sequence $(A)$, a low signal on T1 post-contrast early and delayed sequence $(B, C)$, and a high signal on the diffusion sequence (D). Note that the mass-like area has a high signal on the Apparent Diffusion Coefficient (ADC) MAP (E) rather than a low signal, which is suggestive of response to treatment. B, bladder; V, vagina with gel. implementation of high dose rate (HDR) brachytherapy, 3D imagebased planning, and intensity-modulated radiotherapy. In a study by Vargo et al, ${ }^{11}$ the investigators evaluated patients with endometrial cancer who also had clinical involvement of the cervix with neoadjuvant external beam radiotherapy (45-50.4 Gy in 25-28 fractions) and image-based HDR brachytherapy (5-5.5 Gy times 3-4 fractions) plus chemotherapy followed by extra-fascial hysterectomy performed at a median of 6 weeks after radiotherapy. The authors found that at the time of surgery $91 \%$ of patients had no clinical cervical involvement, $58 \%$ had no pathological cervical involvement, and $24 \%$ had a complete pathologic response. With a median follow-up time of 20 months, they noted a 3-year local control, regional control, distant control, disease-free survival, and overall survival rates of $96 \%, 89 \%, 84 \%, 73 \%$, and $100 \%$, respectively.

As it pertains to the surgical approach, we had a discussion with the patient regarding the option of a minimally invasive approach versus an open approach. The rationale for discussion of the open approach was raised given the results of a recently published trial by Ramirez et al ${ }^{12}$ comparing open versus minimally invasive radical hysterectomy in the setting of cervical cancer. In that trial, the investigators found that the rate of disease-free survival at 4.5 years was $86.0 \%$ with minimally invasive surgery and $96.5 \%$ with open surgery, and that minimally invasive surgery was also associated with a lower rate of overall survival (3-year rate, $93.8 \%$ vs $99.0 \%$; $H R$ for death from any cause 6.00; $95 \% \mathrm{Cl} 1.77$ to 20.30). This study, at least in discussions held in the multidisciplinary conference, raised concerns for performing minimally invasive surgery in patients with endometrial cancer with documented extension to the uterine cervix. When considering results from the prospective randomized trial comparing open versus minimally invasive surgery in patients with endometrial cancer (GOG-LAP2), ${ }^{13}$ interestingly the rate of recurrence was $18.1 \%$ for patients with stage II disease; however, the number of patients was too small to draw any definitive conclusions and the study was not designed to evaluate a difference between the two approaches in this patient population. Therefore, based on discussions with the patient regarding these facts, the patient elected to proceed with the open approach.

In August 2018 the patient underwent a total abdominal hysterectomy and bilateral salpingo-oophorectomy by laparotomy via a low-transverse incision. Findings at surgery revealed a $14 \mathrm{~cm}$ uterus and no evidence of extra-uterine disease. Estimated blood loss was $200 \mathrm{~mL}$. The patient had an uneventful post-operative course and was discharged to home on post-operative day 2 . Final pathology showed an endometrial polyp with complex hyperplasia with atypia. Cervix had evidence of ulceration and chronic inflammation. The patient was last seen and evaluated in April 2019 and was well and without evidence of disease.

\section{CLOSING SUMMARY}

The optimal management of patients with stage II endometrial cancer remains a topic of frequent discussion, and often of significant debate, particularly when considering patients with multiple medical co-morbidities or at high risk for peri-operative complications. In addition, this may become increasingly more challenging when confronted with large and bulky tumors encompassing the entire endometrial cavity and extending to the cervix. Given the low 


\section{Case Studies}

incidence of endometrial cancer with extension to the cervix, there is a paucity of prospective data outlining the ideal management of such patients and conducting a randomized controlled trial may not be feasible.

The decision to proceed with pre-operative combined chemotherapy and radiation was primarily dictated by the patient's obesity and the anticipated risk of peri-operative complications when considering a radical hysterectomy. In addition, there was concern for potential positive margins in the surgical specimen as a result of the extent of local tumor involvement, thus indicating the need for subsequent radiotherapy after radical hysterectomy. Therefore, it is imperative to discuss with the patient that the possible benefits of a radical hysterectomy may need to be weighed against the increased morbidity of the combined approach. Based on available data, neoadjuvant chemoradiotherapy with image-based brachytherapy followed by extra-fascial hysterectomy would appear to be a very reasonable option in such patients.

Contributors CK - Presenter, PTR - Discussant, AJ - Discussant, PB - Radiologist, RB - Pathologist. All contributed to the conception and writing of the manuscript.

Funding The authors have not declared a specific grant for this research from any funding agency in the public, commercial or not-for-profit sectors.

Competing interests None declared.

Patient consent for publication Not required.

Provenance and peer review Commissioned; internally peer reviewed.

\section{REFERENCES}

1. Westin SN, Lacour RA, Urbauer DL, et al. Carcinoma of the lower uterine segment: a newly described association with Lynch syndrome. J Clin Oncol 2008;26:5965-71.
2. Berman ML, Ballon SC, Lagasse LD, et al. Prognosis and treatment of endometrial cancer. Am J Obstet Gynecol 1980;136:679-88.

3. Barwick TD, Rockall AG, Barton DP, et al. Imaging of endometrial adenocarcinoma. Clin Radiol 2006;61:545-55.

4. Nagar H, Dobbs S, McClelland HR, et al. The diagnostic accuracy of magnetic resonance imaging in detecting cervical involvement in endometrial cancer. Gynecol Oncol 2006;103:431-4.

5. Ramirez PT, Frumovitz M, Milam MR, et al. Limited utility of magnetic resonance imaging in determining the primary site of disease in patients with inconclusive endometrial biopsy. Int J Gynecol Cancer 2010;20:1344-9.

6. $\mathrm{He} \mathrm{H}$, Bhosale P, Wei W, et al. MRI is highly specific in determining primary cervical versus endometrial cancer when biopsy results are inconclusive. Clin Radiol 2013;68:1107-13.

7. Park J-Y, Kim EN, Kim D-Y, et al. Comparison of the validity of magnetic resonance imaging and positron emission tomography/ computed tomography in the preoperative evaluation of patients with uterine corpus cancer. Gynecol Oncol 2008;108:486-92.

8. Koh WJ, Abu-Rustum NR, Bean S, et al. NCCN clinical practice guidelines in oncology: Cervical cancer. version 1.2019. Cervical cancer, version 3.2019, NCCN clinical practice guidelines in oncology. J Natl Compr Canc Netw 2019;17:64-84.

9. Ozgul N, Boyraz G, Salman MC, et al. Oncological outcomes of stage II endometrial cancer: a retrospective analysis of 250 cases. Int J Gynecol Cancer 2018;28:161-7.

10. Higgins RV, van Nagell JR, Horn EJ, et al. Preoperative radiation therapy followed by extrafascial hysterectomy in patients with stage II endometrial carcinoma. Cancer 1991;68:1261-4.

11. Vargo JA, Boisen MM, Comerci JT, et al. Neoadjuvant radiotherapy with or without chemotherapy followed by extrafascial hysterectomy for locally advanced endometrial cancer clinically extending to the cervix or parametria. Gynecol Oncol 2014;135:190-5.

12. Ramirez PT, Frumovitz M, Pareja R, et al. Minimally invasive versus abdominal radical hysterectomy for cervical cancer. N Engl J Med 2018;379:1895-904.

13. Walker JL, Piedmonte MR, Spirtos NM, et al. Recurrence and survival after random assignment to laparoscopy versus laparotomy for comprehensive surgical staging of uterine cancer: Gynecologic Oncology Group LAP2 study. J Clin Oncol 2012;30:695-700. 